\title{
Reseracth Sulure \\ Novel Hollow Octagonal Core Photonic Crystal Fiber (PCF) based Cholesterol Sensor
}

\author{
Abdullah Al-Mamun Bulbul \\ Khulna University \\ Etu Podder \\ Khulna University \\ Osama S. Faragallah \\ Taif University \\ Mohammed Baz \\ Taif University \\ Mahmoud M. A. Eid \\ Taif University \\ ahmed nabih zaki rashed ( $\square$ ahmed_733@yahoo.com ) \\ Menoufia University https://orcid.org/0000-0002-5338-1623
}

\section{Research Article}

Keywords: Cholesterol, confinement loss, hollow octagonal core, PCF, sensitivity

Posted Date: July 26th, 2021

DOI: https://doi.org/10.21203/rs.3.rs-596233/v1

License: (c) (i) This work is licensed under a Creative Commons Attribution 4.0 International License.

Read Full License 


\title{
Novel Hollow Octagonal Core Photonic Crystal Fiber (PCF) based Cholesterol Sensor
}

\author{
Abdullah Al-Mamun Bulbul' ${ }^{1}$, Etu Podder ${ }^{1}$, Osama S. Faragallah², Mohammed Baz ${ }^{3}$, \\ Mahmoud M. A. Eid $^{4}$ and Ahmed Nabih Zaki Rashed ${ }^{5, *}$
}

\author{
${ }^{1}$ Electronics and Communication Engineering Discipline, Khulna University, Khulna-9208, Bangladesh \\ Emails: bulbul@ku.ac.bd; e2.120926@gmail.com \\ ${ }^{2}$ Department of Information Technology, College of Computers and Information Technology, \\ Taif University, P.O. Box 11099, Taif 21944, Saudi Arabia \\ Email: o.salah@tu.edu.sa \\ ${ }^{3}$ Department of Computer Engineering, College of Computers and Information Technology, \\ Taif University, P.O. Box 11099, Taif 21944, Saudi Arabia \\ Email:mo.baz@tu.edu.sa \\ ${ }^{4}$ Department of Electrical Engineering, College of Engineering, Taif University, \\ P.O. Box 11099, Taif 21944, Saudi Arabia \\ E-mail: m.elfateh@tu.edu.sa \\ ${ }^{5}$ Electronics and Electrical Communications Engineering Department, \\ Faculty of Electronic Engineering, Menouf 32951, Menoufia University, Egypt \\ "Corresponding Author: E-mail: ahmed_733@yahoo.com
}

\begin{abstract}
Cholesterol, also known as the pulpy fat of human body, may cause the body vulnerable to heart malady by filling bloodvessel with fatty substances. This urges a precise, simple, and effective method of cholesterol detection. Considering this, a novel hollow equilateral-octagon core PCF model has been presented as a cholesterol sensor. The sensor is modeled and simulated using finite element method (FEM). Multiple performance metrics suggests the effectiveness of the proposed sensor model. The relative sensitivity attained for the model is $92.34 \%$ at optimum frequency (OF), 3.6THz. Besides, the model shows ultra-lower confinement loss of $3.77 \times 10^{-18} \mathrm{~cm}^{-1}$ at $\mathrm{OF}$ along with standard values for other performance metrics. In addition, the feasible implementation of the model by exercising existing fabrication strategies ensures the practicability of the proposed sensor.
\end{abstract}

Keywords: Cholesterol; confinement loss; hollow octagonal core; PCF; sensitivity.

\section{Introduction}

Cholesterol is the soft and pulpy fat in our body. It is a very worthy element of the human body since cholesterol assists to preserve the structural integrity as well as it carries on the fluidity of cell membranes. Besides, it also controls the production of Vitamin D in our body. The major source of cholesterol in the human body is egg, milk, meat, etc. Therefore, the presence of an immoderate amount of cholesterol in our body can lead to heart disease, high blood pressure, or stroke. So, to understand the level of cholesterol in our body we need to check up regularly from age 35 or earlier for men and age 45 or earlier for women. Cholesterol can be good or bad for our health. Because of the presence of Low-density lipoprotein (LDL) cholesterol in our body can lead to heart attack and stroke. So, LDL cholesterol is bad for us. Again, the presence of High-density lipoprotein (HDL) cholesterol in our body helps to remove LDL cholesterol. For this reason, HDL cholesterol is good for human health. Usually, the total amount of cholesterol in human blood is less than 200 milligrams per deciliter while the amount of LDL is around 70 to 130 milligrams per deciliter and the amount of HDL is around 40 to 60 milligrams per deciliter. For LDL cholesterol, the lower amount is better and for HDL cholesterol, the higher amount is better. The main way of measuring the amount of cholesterol in the human body is a blood test. Hence, an efficient technique of cholesterol detection is very urgent.

So far, several methods have been reported for detecting biochemical, for example, Chromatographic [1], voltametric [2], electrometric [3], etc. However, these sensing methods possess some drawbacks, for instance, long investigation time, the measurement procedure is complex, expert manpower (operating assistant) is required, etc. For these reasons, still researchers are finding an efficient way to sense biochemicals. For the last two decades, researchers have realized that the photonic crystal fiber (PCF) can provide efficient outcomes, especially for sensing applications. Recently, liquid sensing, gas sensing, and chemical sensing fields are explored by different kinds of PCF sensors [4-9]. Besides, the intensity measurement strategy of EM waves has also made the PCF capable of operating remotely.

The research related to chemical sensing especially in $\mathrm{THz}$ region has attained the attention of researchers, engineers, academia, industry, etc. THz sensing got introduced by Jepsen et al. [10,11] in early 2017 where they perform chemical sensing. Recently, a heptagonal shaped PCF is proposed by Hasan et. al. [12] for sensing water, ethanol, and benzene where they found the maximum sensitivity for benzene is around $63.04 \%$. The same experiment is done by Kanmani et. al. [13] for different PCF structure, where they found around $77.08 \%$ sensitivity for benzene. Ahmed et al. [14] presented a sensor for blood component detection while they found the sensitivity of approximately $80.93 \%$ for the Red Blood Cells. Islam et al. [15] reported a complex 
sensor structure for chemical detection where they achieved a sensitivity of approximately $86 \%$. Hossain et al. [16] have reported a rectangular hollow-core PCF sensor to identify the bane chemicals and they achieved a sensitivity of almost $94.4 \%$. Therefore, there is still a chance to develop the performance of chemical sensing using PCF in the THz region.

In this paper, we have proposed a PCF model for cholesterol detection in the Terahertz regime. The proposed model affords the relative sensitivity of around $98 \%$ and we have also numerically investigated several significant optical properties, for instance, confinement loss, effective area, EML, etc. The proposed model offers better sensing characteristics than some of the recently proposed sensor models (Terahertz sensor) in sensing applications [12-20].

\section{Designing Strategies}

The proposed cholesterol sensor has been modeled using hollow core PCF (HC-PCF). The reason behind this hollow core is its inherent advantageous features over porous core PCF (PC-PCF). HC-PCF provides space for increased quantity of analytes at the core region. Besides, improved collaboration between the core material and transitory field is observed in this type of PCF since guiding mode is highly confined in the core region and the analyte injected into the core region gets more interacted with the light in this type of PCF [21,22]. In addition, HC-PCF hardly suffers from effective material loss (EML) since only samples are injected into the core region. This type of sample injection also ensures the presence of higher portion of light in the core region. As a whole, HC-PCF offers greater sensitivity compared to PC-PCF.

The structure of the proposed PCF based sensor has been modeled and analyzed using finite element method (FEM). This offers platform for both designing and simulation of PCF model. The software is founded on finite element analysis that is capable to handle numerous simultaneous electromagnetic (EM) modes, operate with numerous light-sources, spontaneously generate analogous partial differential equations, and provide numerical results [23]. Additionally, FEM can efficiently handle many composite substance features as well as it can collaborate with Matlab software [24].

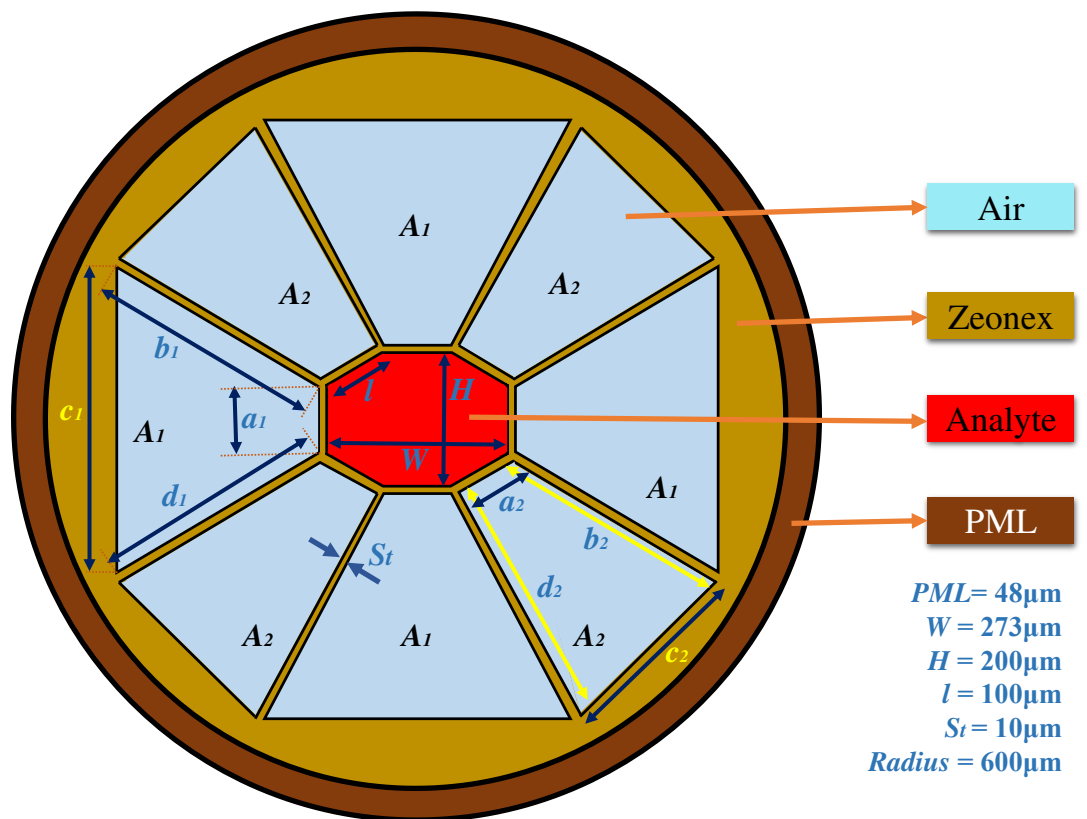

Fig. 1. The transversal schematic diagram for the anticipated sensor model.

The transversal schematic diagram for the anticipated sensor model has been represented in Fig. 1. The core region of the sensor model consists of a hollow equilateral-octagon where analytes should be injected to be sensed. The length of each side of the octagon, denoted by $l$ in the figure, is $100 \mu \mathrm{m}$. The width and height of the octagon are $273 \mu \mathrm{m}$ and $200 \mu \mathrm{m}$ respectively. The total area covered by the octagonal core is $45950 \mu \mathrm{m}^{2}$. The cladding region for the proposed sensor geometry is formed by the asymmetric organization of eight quadrilateral hollow structures. Two different sets of quadrilateral structures (as represented by $A_{1}$ and $A_{2}$ in the model) construct the cladding region. The four sides of $A_{1}$ and $A_{2}$ type of quadrilateral are indicated by $\mathrm{a}_{1}, \mathrm{~b}_{1}, \mathrm{c}_{1}$, $\mathrm{d}_{1}$, and $\mathrm{a}_{2}, \mathrm{~b}_{2}, \mathrm{c}_{2}, \mathrm{~d}_{2}$ respectively. The lengths of $\mathrm{a}_{1}, \mathrm{~b}_{1}, \mathrm{c}_{1}$, and $\mathrm{d}_{1}$ are about $100,350,457$, and $350 \mu \mathrm{m}$ respectively. Each $A_{1}$ covers an area of around $83966 \mu \mathrm{m}^{2}$. Whereas, the values of $\mathrm{a}_{2}, \mathrm{~b}_{2}, \mathrm{c}_{2}$, and $\mathrm{d}_{2}$ are approximately $95,348,278$, and $376 \mu \mathrm{m}$ respectively and the total area covered by a single $A_{2}$ quadrilateral structure is nearly $64453 \mu \mathrm{m}^{2}$. The gap between two consecutive blocks, known as strut and denoted by $S_{t}$ in Fig. 1 , is chosen to be $10 \mu \mathrm{m}$. The radius of the modeled sensor is $600 \mu \mathrm{m}$. To avoid ecological interferences, a layer in the outermost region is introduced which is known as a perfectly matched layer (PML). PML resists the departing waves from the fiber and thereby, it acts as a reflection-resistant cover [25]. Here, the thickness of PML is set as 8\% of the radius of the proposed model and the value of it becomes $48 \mu \mathrm{m}$. Zeonex is accommodated as the background substance for this model as it maintains constant value for the refractive index $(\mathrm{RI}=1.53)$ in $\mathrm{THz}$ regime, minimized amount of substance 
absorption loss, and dispersion, moisture insensitiveness, etc. [16]. Besides, zeonex exhibits an increased amount of chemical restiveness as well as greater crystal transition temperature that helps to fabricate the fiber flexibly. The above reason influences us to elect zeonex over topas, silica, and other fiber materials. To present a comparative analysis we have varied the core region of the optimum model by $\pm 25 \%$ and thereby we found two additional model structure named as optimum model $-25 \%$ and optimum model $+25 \%$. The core region of each model is injected by cholesterol and the simulation is performed. Again, the optimum sensor model is also simulated to explore the performance of the model in detecting blood and water.

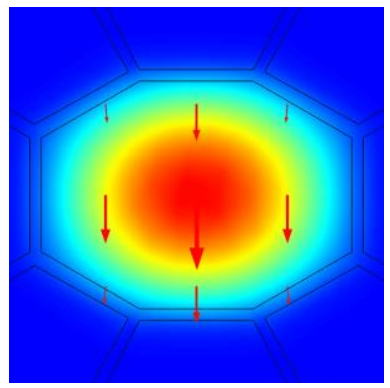

(a)

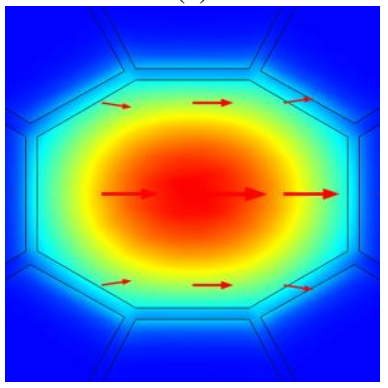

(d)

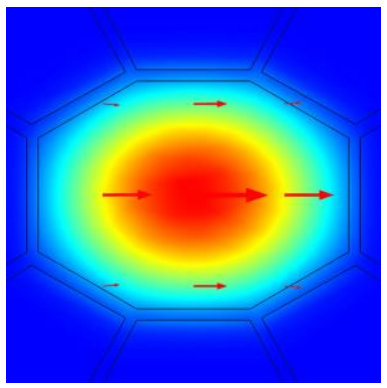

(b)

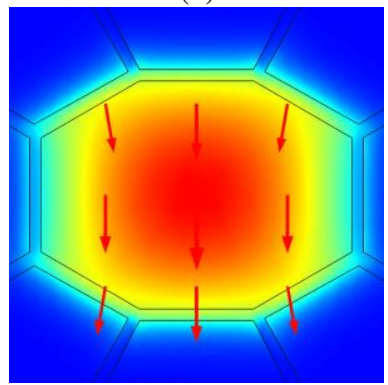

(e)

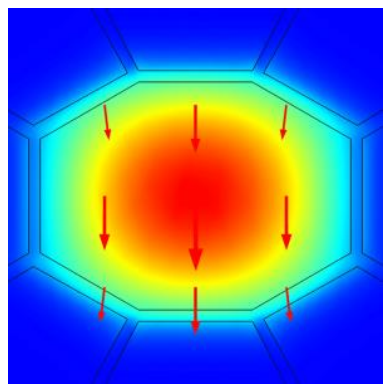

(c)

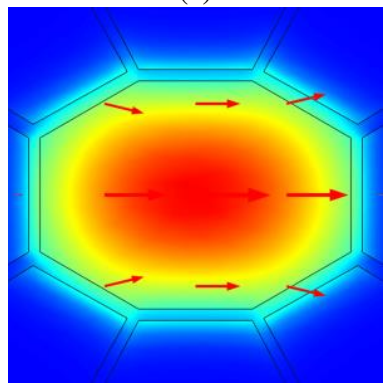

(f)

Fig. 2. EM wave propagation through the core of optimum model in $x$ and $y$ direction for cholesterol [(a),(b)], blood [(c),(d)], and water [(e),(f)].

\section{Method of Analysis and Simulation Results}

Simulation of the anticipated sensor model has been performed for a wide range of frequency bands ranging from 2.0 to 4.0THz. The EM wave propagation through the core of the optimum model in both $x$ and $y$ propagation directions for three different samples namely the cholesterol, blood, and water is shown in Fig. 2. We have injected the samples, i.e., cholesterol, blood, and water, through the core once at a time and simulated the model separately.

To evaluate how effectively the proposed sensor will detect the samples, we have estimated the relative sensitivity $\left(R_{S}\right)$ for the proposed model in each case of sample injection. $R_{S}$ is the prime sensing metric that specifies the presence and quantity of a specific sample in the core. It is estimated using the following equation (1) [16,17].

$$
R_{S}=\frac{\eta_{\text {sample }}}{\eta_{\text {effective }}} \times B
$$

Here, $\eta_{\text {sample }}$ indicates the RI of anticipated samples, which is $1.525,1.376$, and 1.33 for cholesterol, blood, and water respectively. Besides, $\eta_{\text {effective }}$ defines the RI of effective mode and $B$ indicates the extent of the entire power of injected light present at the core. $B$ is evaluated using equation (2) $[17,26]$.

$$
B=\frac{\int \operatorname{Re}\left[E_{x} H_{y}-E_{y} H_{x}\right] \mathrm{dx} \cdot \mathrm{dy}}{\int \operatorname{Re}\left[E_{x} H_{y}-E_{y} H_{x}\right] \mathrm{dx} \cdot \mathrm{dy}} \times 100 \%
$$

Here, $E$ and $H$ represent the electric and magnetic field constituents, and the subscript $x$ and $y$ define the transversal and tangential constituents respectively. 


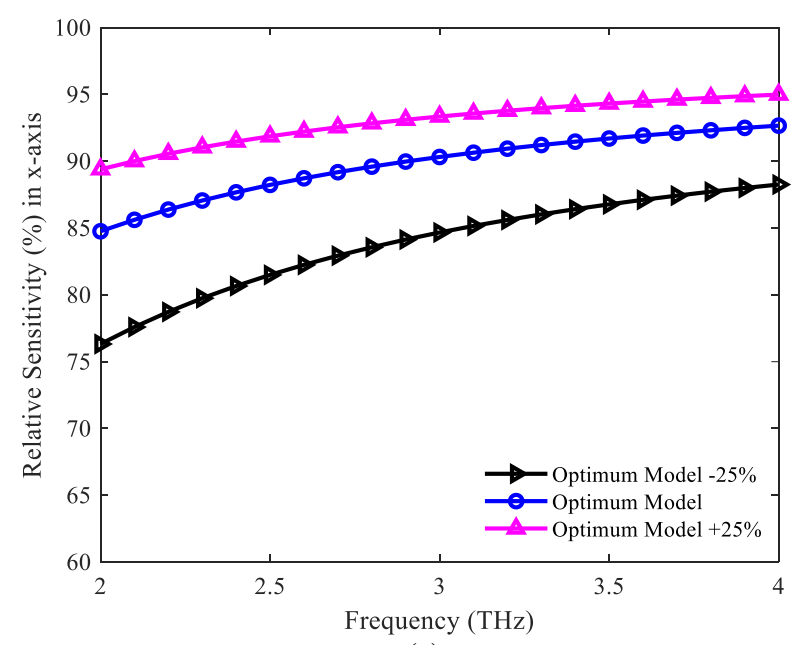

(a)

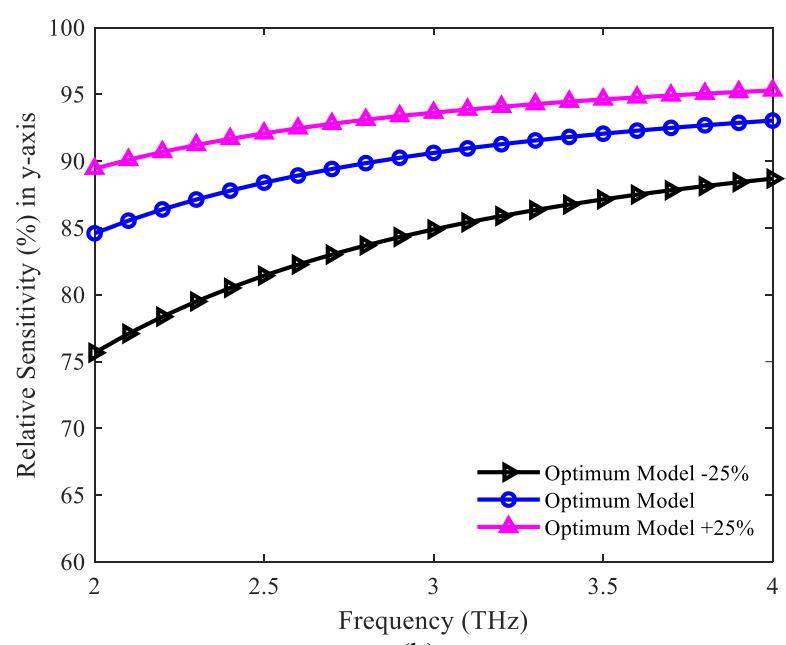

(b)

Fig. 3. Comparison of relative sensitivity in the (a) $x$-axis and (b) $y$-axis for three different models.

The variation of relative sensitivity with the increase in frequency for the three different models namely, optimum-25\%, optimum, and optimum $+25 \%$ in $x$ and $y$ polarization direction are shown in Fig. 3(a) and Fig. 3(b) respectively. As found from Fig. 3(a), the values of relative sensitivity in the $x$-axis for optimum- $25 \%$, optimum, and optimum $+25 \%$ are $87.12 \%, 91.93 \%$, and 94.47\% respectively at the optimum frequency (OF) of $3.6 \mathrm{THz}$. Besides, Fig. 3(b) reflects that the relative sensitivity in the $y$ axis for optimum $-25 \%$, optimum, and optimum $+25 \%$ are $87.59 \%, 92.34 \%$, and $94.81 \%$ respectively at the OF. The optimum, and optimum $+25 \%$ models show dominant sensitivity compared to the optimum- $-25 \%$ model.

Fig. 4(a) and Fig. 4(b) represent the relative sensitivity in the $x$ and $y$-axis respectively while detecting cholesterol, blood, and water. The relative sensitivity in the $x$-axis for water, cholesterol, and blood at the OF are $80.36 \%, 91.93 \%$, and $84.65 \%$, respectively. The relative sensitivity in the $y$-axis for water, cholesterol, and blood are $82.46 \%, 92.34 \%$, and $86.11 \%$, respectively at the OF. In both directions, the optimum sensor model exhibits dominant relative sensitivity in detecting cholesterol.

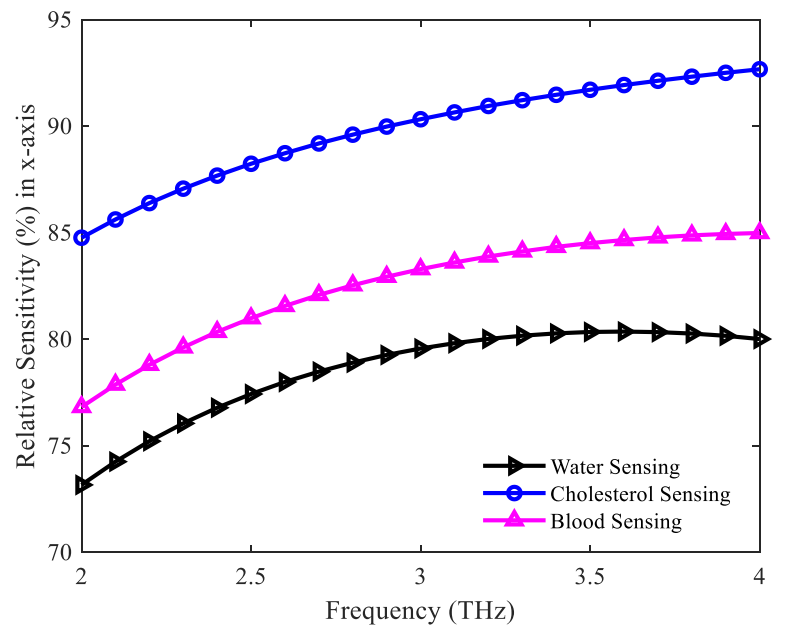

(a)

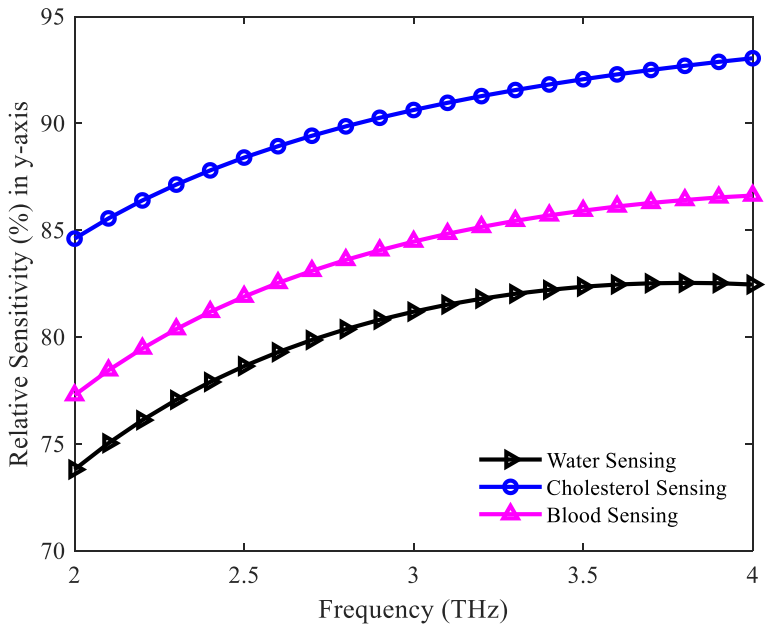

(b)

Fig. 4. Comparison of relative sensitivity in the (a) $x$-axis and (b) $y$-axis for three different samples.

Birefringence is the degree of asymmetry between core and cladding air holes. It maintains the polarization attributes. It is the absolute deviation between the RI for $x$-polarization $\left(\eta_{x}\right)$ and $y$-polarization $\left(\eta_{x}\right)$ mode as shown in equation (3) [17].

$$
\text { Birefringe nce }=\left|\eta_{x}-\eta_{y}\right|
$$

The comparison of birefringence as a function of frequency for the three models is shown in Fig. 5(a). The value of birefringence for optimum $-25 \%$, optimum, and optimum $+25 \%$ are $0.0014,0.0007$, and 0.0005 respectively at the OF. The value of birefringence for the optimum $+25 \%$ is lowest compared to the other two and hence, this model is poorly birefringent. 


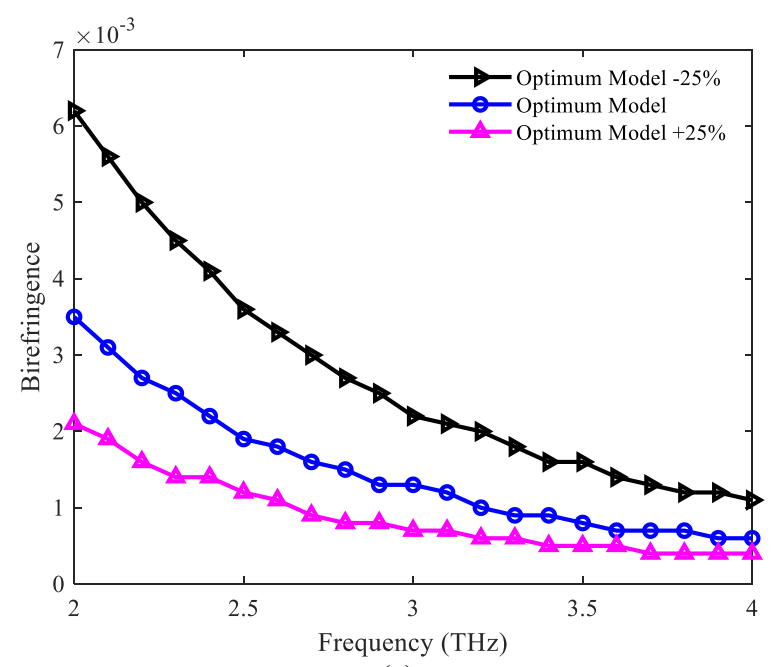

(a)

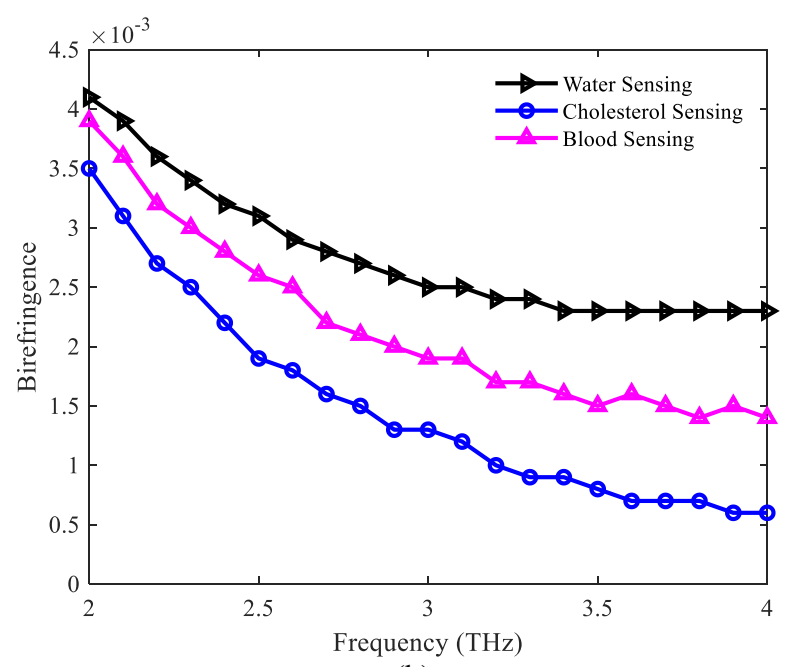

(b)

Fig. 5. Variation of birefringence as a function of frequency for (a) three models and (b) three different samples.

Fig. 5(b) displays the birefringence of the optimum model for three different samples. The values for birefringence while detecting water and blood are higher but they suffer from lower relative sensitivity. Another key performance metrics of PCF is the confinement loss $\left(C_{\text {Loss }}\right)$, which indicates the amount of light leakage in the cladding regions. Indirectly, it reflects how the incoming light is confined in the core region. It is evaluated as a function of operating frequency $(f)$, light speed $(c)$, and the imaginary portion of $\eta_{\text {effective }}$ as shown in the following equation (4) [17,27].

$$
C_{\text {Loss }}=\frac{4 \pi f}{c} \times \operatorname{Im}\left[\eta_{\text {effective }}\right] \mathrm{cm}^{1}
$$

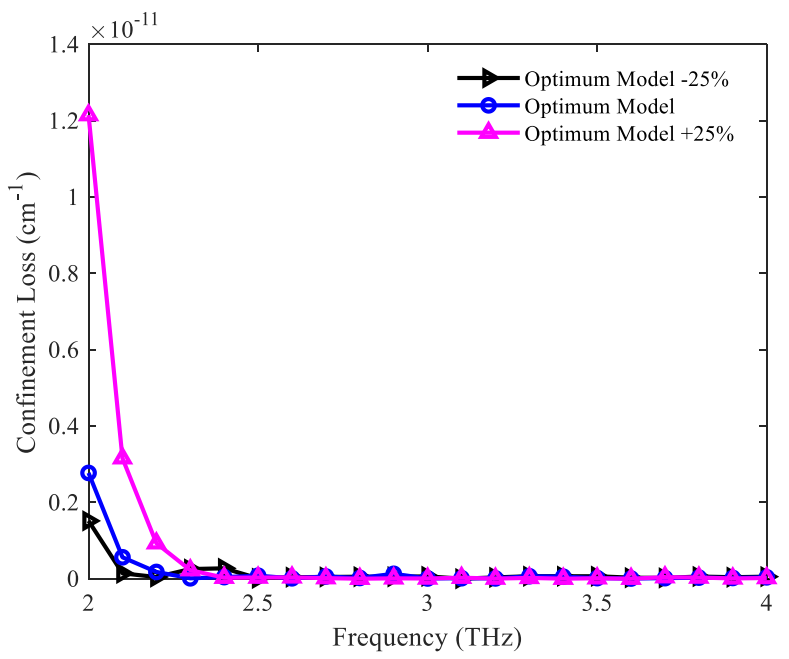

(a)

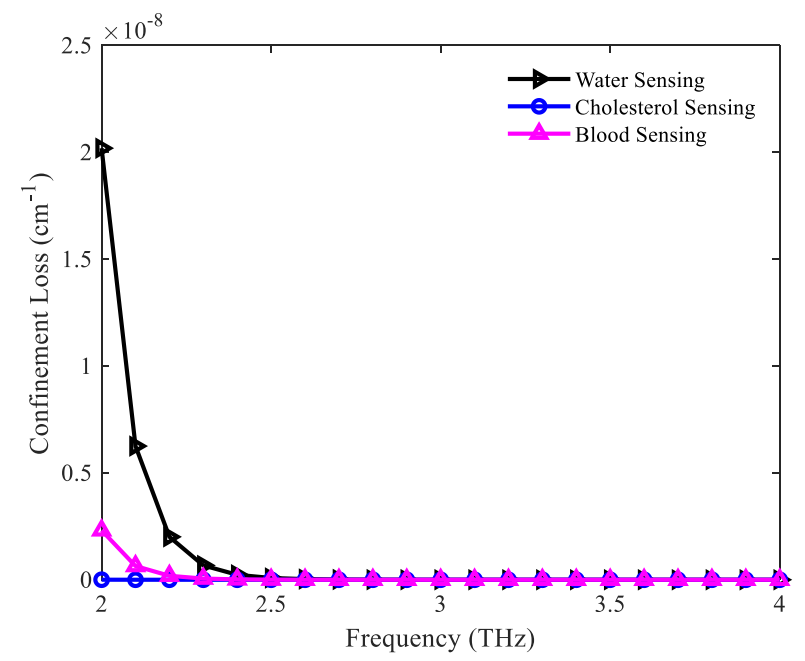

(b)

Fig. 6. Representation of confinement loss for (a) three different models and (b) three different samples.

Fig. 6(a) and Fig. 6(b) represent the amount of confinement loss for three different models and three different samples respectively. The value of $C_{\text {Loss }}$ for the optimum- $25 \%$, optimum, and optimum $+25 \%$ model are $1.89 \times 10^{-17}, 3.77 \times 10^{-18}$, and $1.37 \times 10^{-18} \mathrm{~cm}^{-1}$ respectively at the OF. On the other hand, the values of $C_{\text {Loss }}$ for water, cholesterol and blood at the OF are $9.09 \times 10^{-18}, 3.77 \times 10^{-18}$, and $8.71 \times 10^{-18} \mathrm{~cm}^{-1}$, respectively. The value of $C_{\text {Loss }}$ for the optimum model in detecting cholesterol is dominant as it is the lowest. Lower values of $C_{\text {Loss }}$ indicate higher signal confinement in the core region which results in a very negligible amount of coupling and scattering loss. The EML is measured for the proposed model as it appears when light is engaged by zeonex. Following equation (5) is used to calculate EML $\left(E M_{\text {Loss }}\right)[17,16]$.

$$
\mathrm{EM}_{\text {Loss }}=\sqrt{\frac{\varepsilon_{0}}{\mu_{0}}} \times \frac{\int \eta_{\text {bulk }}|E|^{2} \alpha_{\text {bulk }} \mathrm{dA}}{\left|\int \frac{1}{2}(E \times H) z \mathrm{dA}\right|}, \mathrm{cm}^{-1}
$$

Where, $\varepsilon_{0}, \mu_{0}$, and $H^{*}$ outlines the permittivity, permeability, and transverse magnetic field. $\alpha_{b u l k}$ and $\eta_{b u l k}$ symbolize the absorption loss and RI of bulk material respectively. 


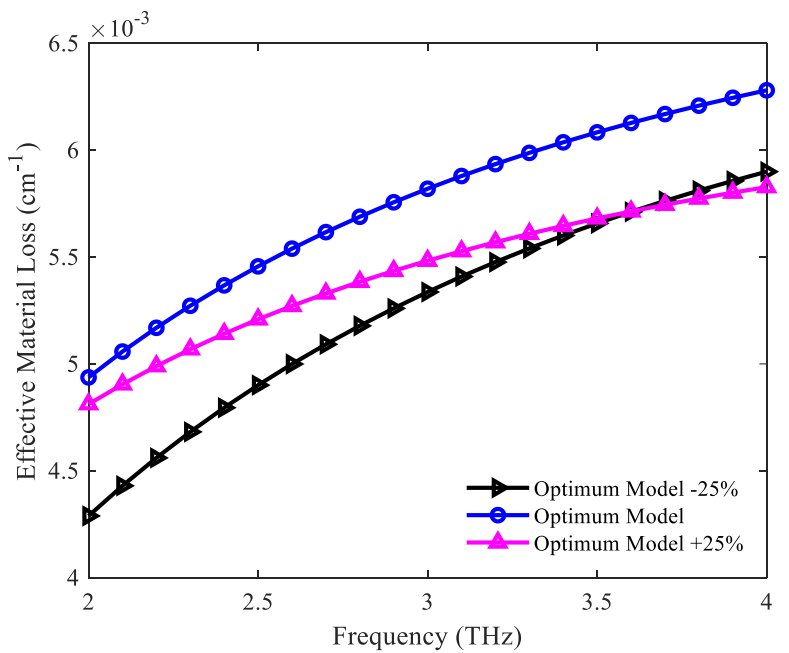

(a)

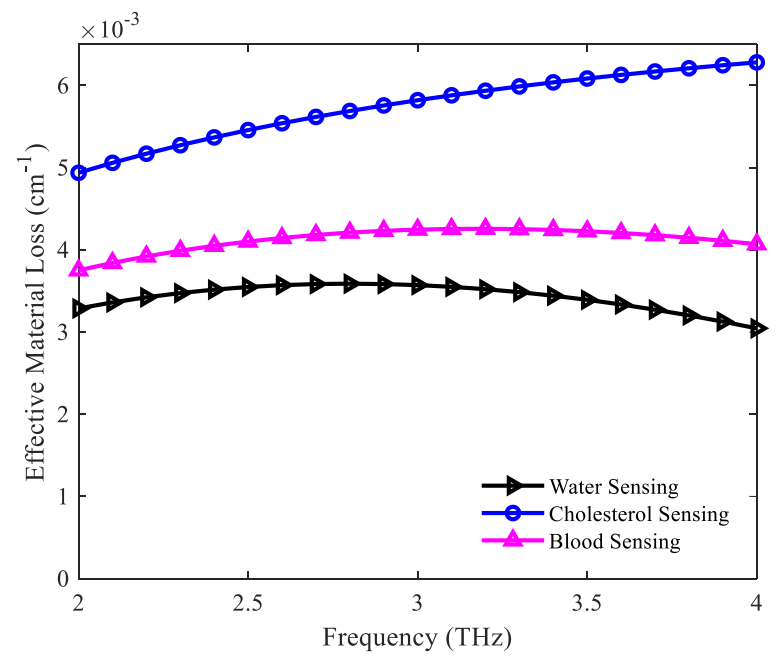

(b)

Fig. 7. EML for (a) three different models and (b) three different samples in the THz regime.

Fig. 7(a) and Fig. 7(b) picture the EML as a function of frequency for three different models and three different samples respectively. As demonstrated from Fig. 7(a), the values of EML for three different models are nearly the same at the OF. But the value of EML is a little bit higher for cholesterol detection compared to the other two sample detection at the OF.

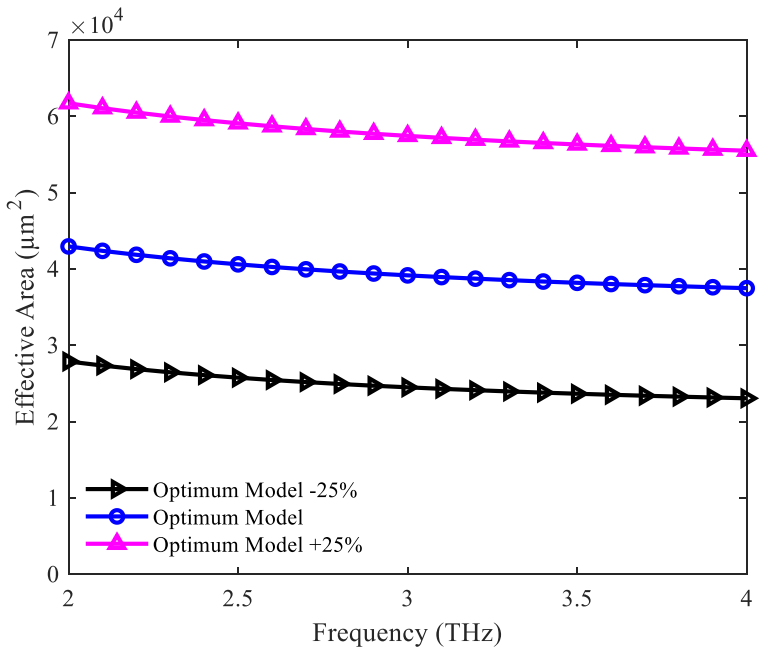

(a)

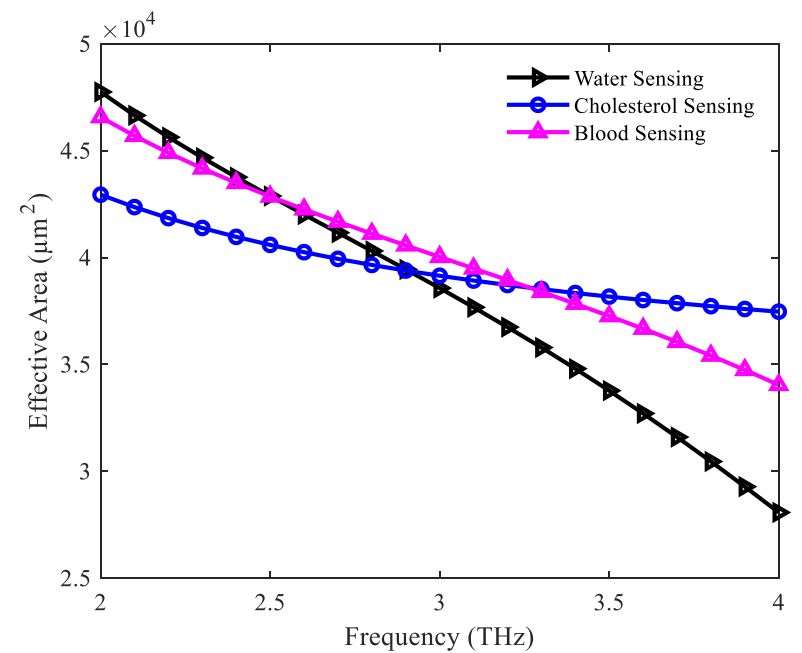

(b)

Fig. 8. Representation of the effective area for (a) three different models and (b) three different samples.

Effective area $\left(E_{\text {Area }}\right)$ states the true sensing area of a PCF. It is evaluated using equation (6) [27,28].

$$
E_{\text {Area }}=\frac{\left(\iint\left|E^{2}\right| d x . d y\right)^{2}}{\iint\left|E^{4}\right| d x . d y}, \mu m^{2}
$$

Fig. 8(a) demonstrates the variation of effective area for three different models where the optimum $+25 \%$ model exhibits the highest value for $E_{\text {Area }}$ at the $\mathrm{OF}$ i.e., $56112 \mu \mathrm{m}^{2}$. The highest value signifies signal spreading or dispersion in a wider region which is not expected in chemical sensing applications. Hence, the optimum $+25 \%$ model is not an acceptable one compared to the other two models. Fig. 8(b) represents the $E_{\text {Area }}$ for sensing three different samples by the optimum model. The values for $E_{\text {Area }}$ are adjacent to each other for all three samples at the OF. Another key optical parameter is the numerical aperture $\left(N_{\text {Aperture }}\right)$ which quantifies the signal compiling skill of any fiber. A higher value of $N_{\text {Aperture }}$ is expected for chemical detection applications and the value for $N_{\text {Aperture }}$ is evaluated by the equation (7) [15].

$$
N_{\text {Aperture }}=\left(1+\pi E_{\text {Area }} f^{2} c^{-2}\right)^{-1 / 2}
$$




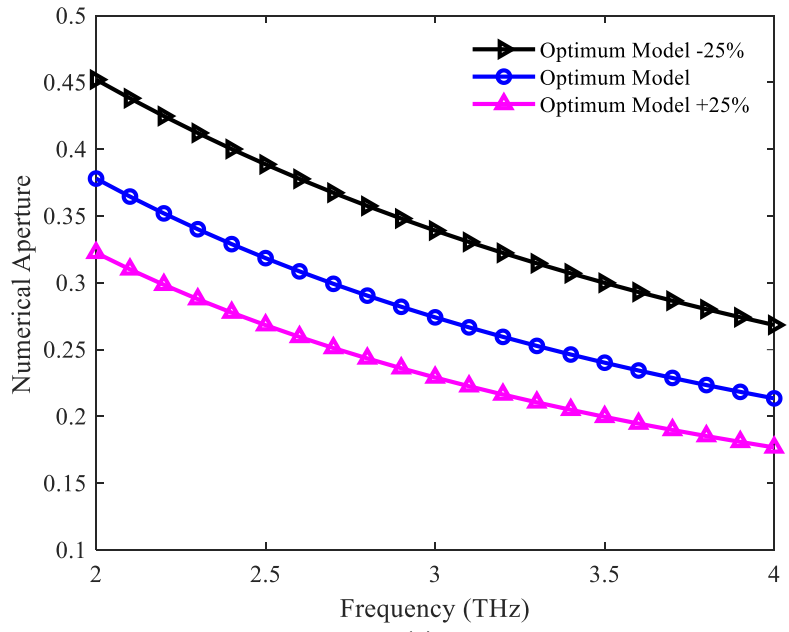

(a)

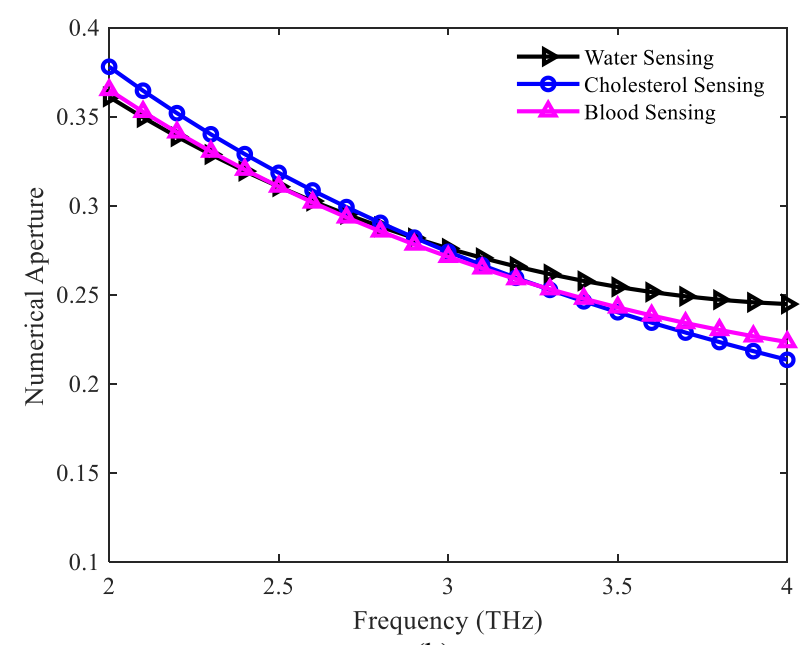

(b)

Fig. 9. Variation of numerical aperture for (a) three different models and (b) three different analytes.

Fig. 9(a) represents the $N_{\text {Aperture }}$ for three different models where optimum-25\% and optimum model show dominant value compared to optimum $+25 \%$ model. The value of $N_{\text {Aperture }}$ attained for the optimum model is 0.234 at the OF. Fig. 9 (b) reveals the $N_{\text {Aperture }}$ for detecting three different samples. The values of $N_{\text {Aperture }}$ are adjacent for all the three analytes at the OF.

\section{Summery and Fabrication Possibilities}

Table 1 represents the comparison of the optical parameters for three different models. The optimum model shows comparably higher sensitivity in both $\mathrm{x}$ and $\mathrm{y}$-axis, higher numerical aperture, and standard birefringence along with lower values for both EML and confinement loss compared to the other models. Hence, the optimum model is selected as the proposed cholesterol sensor.

Table 1. Tabular representation of optical parameters for three different models at the OF.

\begin{tabular}{|c|c|c|c|}
\hline Parameters & Optimum Model -25\% & Optimum Model & Optimum Model +25\% \\
\hline Effective Area & $23510 \mu \mathrm{m}^{2}$ & $38017 \mu \mathrm{m}^{2}$ & $56112 \mu \mathrm{m}^{2}$ \\
\hline Confinement Loss & $1.89 \times 10^{-17} \mathrm{~cm}^{-1}$ & $3.77 \times 10^{-18} \mathrm{~cm}^{-1}$ & $1.37 \times 10^{-18} \mathrm{~cm}^{-1}$ \\
\hline Numerical Aperture & 0.29 & 0.234 & 0.19 \\
\hline Sensitivity in $\boldsymbol{x}$-axis & $87.12 \%$ & $91.93 \%$ & $94.47 \%$ \\
\hline EML & $0.0058 \mathrm{~cm}^{-1}$ & $0.0062 \mathrm{~cm}^{-1}$ & $0.0058 \mathrm{~cm}^{-1}$ \\
\hline Birefringence & 0.0014 & 0.0007 & 0.0005 \\
\hline Sensitivity in $\boldsymbol{y}$-axis & $87.59 \%$ & $92.34 \%$ & $94.81 \%$ \\
\hline
\end{tabular}

Table 2 is the comparative representation of the optimum model while detecting water, cholesterol, and blood. The proposed model maintains a higher level of sensitivity in detecting cholesterol among the three samples. Table 3 is the tabular comparison of the proposed sensor with the existing PCF based sensors. The proposed sensor is superior to the other sensors as it shows the lowest confinement loss, lower EML, higher $N_{\text {Aperture }}$, standard effective area, and more importantly higher relative sensitivity.

Table 2. Tabular representation of optical parameters of the optimum model for three different samples at the OF.

\begin{tabular}{|c|c|c|c|}
\hline Parameters & Water & Cholesterol & Blood \\
\hline Effective Area & $32710 \mu \mathrm{m}^{2}$ & $38017 \mu \mathrm{m}^{2}$ & $36674 \mu \mathrm{m}^{2}$ \\
\hline Confinement Loss & $9.09 \times 10^{-18} \mathrm{~cm}^{-1}$ & $3.77 \times 10^{-18} \mathrm{~cm}^{-1}$ & $8.71 \times 10^{-18} \mathrm{~cm}^{-1}$ \\
\hline Numerical Aperture & 0.252 & 0.234 & 0.238 \\
\hline Sensitivity in $\boldsymbol{x}$-axis & $80.36 \%$ & $91.93 \%$ & $84.65 \%$ \\
\hline EML & $0.0034 \mathrm{~cm}^{-1}$ & $0.0062 \mathrm{~cm}^{-1}$ & $0.0043 \mathrm{~cm}^{-1}$ \\
\hline Birefringence & 0.0023 & 0.0007 & 0.0016 \\
\hline Sensitivity in $\boldsymbol{y}$-axis & $82.46 \%$ & $92.34 \%$ & $86.11 \%$ \\
\hline
\end{tabular}


Table 3. Comparative analysis of the proposed sensor with the existing PCF based sensors.

\begin{tabular}{|c|c|c|c|c|c|c|c|}
\hline Analytes & $\begin{array}{c}\mathbf{O F} \\
(\mathrm{THz})\end{array}$ & $\begin{array}{c}\boldsymbol{E}_{\text {Area }} \\
\left(\mu \mathrm{m}^{2}\right)\end{array}$ & $\begin{array}{c}\boldsymbol{C}_{\text {Loss }} \\
\left(\mathrm{cm}^{-1}\right)\end{array}$ & $\boldsymbol{N}_{\text {Aperture }}$ & $\begin{array}{c}\boldsymbol{E M}_{\text {Loss }} \\
\left(\mathrm{cm}^{-1}\right)\end{array}$ & Birefringence & $\begin{array}{c}\text { Sensitivity } \\
(\boldsymbol{\%})\end{array}$ \\
\hline Alcohol [29] & 225.6 & - & $7.55 \times 10^{-14}$ & - & - & - & 67.66 \\
\hline Hemoglobin [14] & 1.5 & $1.5 \times 10^{5}$ & $1.99 \times 10^{-14}$ & - & - & - & 80.56 \\
\hline Ethanol [12] & 1.0 & $1.51 \times 10^{5}$ & $2.30 \times 10^{-3}$ & - & - & - & $61.05 \%$ \\
\hline Formalin [17] & 1.8 & 97657 & $2.8 \times 10^{-11}$ & - & 0.0048 & & 77.71 \\
\hline Proposed Model & $\mathbf{3 . 6}$ & $\mathbf{3 8 0 1 7}$ & $\mathbf{3 . 7 7 \times 1 0 ^ { - 1 8 }}$ & $\mathbf{0 . 2 3 4}$ & $\mathbf{0 . 0 0 6 2}$ & $\mathbf{0 . 0 0 0 7}$ & $\mathbf{9 2 . 3 4}$ \\
\hline
\end{tabular}

Flexible fabrication is the prime challenging task for any new PCF sensor. A numeral of fabrication strategies has been evolved so far such as stacking, extrusion, stack, and draw (S\&D), sol-gel, 3D printing, etc. [30-34]. S\&D strategy may be practiced for fruitful fabrication for our proposed model as this strategy has already been practiced successfully to fabricate multiple PCF models. Besides, the S\&D strategy is also capable to fabricate multiple variants of suspended microstructure fibers [35]. In addition to the above method, 3D printing is a flexible strategy that is capable to fabricate diverse organizations of holes in PCF models [33]. Multiple researchers have already exercised this method to fabricate versatile models [36, 33]. So the use of a 3D printing strategy will also result in a flexible fabrication of our proposed model.

\section{Conclusion}

The presented HC-PCF with the asymmetrical organization of air holes and zeonex as the fiber material is anticipated as a sensor for cholesterol operating at THz frequency regime. Multiple performance metrics have been evaluated to measure the effectiveness of this proposed model as a cholesterol sensor. The proposed sensor model exhibits higher sensitivity of about $92.34 \%$ at the OF in detecting cholesterol. Besides, the proposed model shows a very much negligible amount of confinement loss of $3.77 \times 10^{-18} \mathrm{~cm}^{-1}$ at the OF. Also, the proposed model maintains lower EML, denser effective area, higher numerical aperture, and standard birefringence. Furthermore, the likelihood of fabrication by practicing existing strategies enhances the effectiveness of the proposed model and will introduce its exercise as a chemical-sensor or biosensor.

Acknowledgements: This study was funded by the Deanship of Scientific Research, Taif University Researchers Supporting Project number (TURSP-2020/08), Taif University, Taif, Saudi Arabia.

Conflict of Interest Statement: The authors declare that they have no competing interests.

\section{REFERENCES}

1. Jaszczak E, Ruman M, Narkowicz S, Namieśnik J, Polkowska Ż (2017) Development of an analytical protocol for determination of cyanide in human biological samples based on application of ion chromatography with pulsed amperometric detection. Journal of analytical methods in chemistry 2017

2. Wu J, Wang L, Wang Q, Zou L, Ye B (2016) The novel voltammetric method for determination of hesperetin based on a sensitive electrochemical sensor. Talanta 150:61-70

3. Timofeyenko YG, Rosentreter JJ, Mayo S (2007) Piezoelectric quartz crystal microbalance sensor for trace aqueous cyanide ion determination. Analytical chemistry 79 (1):251-255

4. Buczynski R (2004) Photonic crystal fibers. Acta Physica Polonica Series A 106 (2):141-168

5. Issa NA, van Eijkelenborg MA, Fellew M, Cox F, Henry G, Large MC (2004) Fabrication and study of microstructured optical fibers with elliptical holes. Optics letters 29 (12):1336-1338

6. Pawar AY, Sonawane DD, Erande KB, Derle DV (2013) Terahertz technology and its applications. Drug invention today 5 (2):157-163

7. Podder E, Hossain MB, Jibon RH, Bulbul AA-M, Mondal HS (2019) Chemical sensing through photonic crystal fiber: sulfuric acid detection. Frontiers of Optoelectronics 12 (4):372-381

8. Ademgil H, Haxha S (2015) PCF based sensor with high sensitivity, high birefringence and low confinement losses for liquid analyte sensing applications. Sensors 15 (12):31833-31842

9. Yakasai I, Abas PE, Kaijage SF, Caesarendra W, Begum F Proposal for a quad-elliptical photonic crystal fiber for terahertz wave guidance and sensing chemical warfare liquids. In: Photonics, 2019. vol 3. Multidisciplinary Digital Publishing Institute, $\mathrm{p} 78$

10. Jepsen PU, Møller U, Merbold H (2007) Investigation of aqueous alcohol and sugar solutions with reflection terahertz timedomain spectroscopy. Optics Express 15 (22):14717-14737

11. Jepsen PU, Jensen JK, Møller U (2008) Characterization of aqueous alcohol solutions in bottles with THz reflection spectroscopy. Optics Express 16 (13):9318-9331 
12. Hasan MM, Sen S, Rana MJ, Paul BK, Habib MA, Daiyan GM, Ahmed K Heptagonal Photonic Crystal Fiber Based Chemical Sensor in THz Regime. In: 2019 Joint 8th International Conference on Informatics, Electronics \& Vision (ICIEV) and 2019 3rd International Conference on Imaging, Vision \& Pattern Recognition (icIVPR), 2019. IEEE, pp 40-44

13. Kanmani R, Ahmed K, Roy S, Ahmed F, Paul BK, Rajan MM (2019) The performance of hosting and core materials for slotted core Q-PCF in terahertz spectrum. Optik 194:163084

14. Ahmed K, Ahmed F, Roy S, Paul BK, Aktar MN, Vigneswaran D, Islam MS (2019) Refractive index-based blood components sensing in terahertz spectrum. IEEE Sensors Journal 19 (9):3368-3375

15. Islam MS, Sultana J, Ahmed K, Islam MR, Dinovitser A, Ng BW-H, Abbott D (2017) A novel approach for spectroscopic chemical identification using photonic crystal fiber in the terahertz regime. IEEE Sensors Journal 18 (2):575-582

16. Hossain MB, Podder E, Bulbul AA-M, Mondal HS (2020) Bane chemicals detection through photonic crystal fiber in THz regime. Optical Fiber Technology 54:102102

17. Abdullah Al-Mamun B, Rayhan Habib J, Sumon Kumar D, Tonmoy R, Md. Avijit S, Md. Bellal H (2020) PCF Based Formalin Detection by Exploring the Optical Properties in THz Regime. Nanoscience \& Nanotechnology-Asia 10:1-8. doi:http://dx.doi.org/10.2174/2210681210999200525171303

18. Abdullah Al-Mamun B, Md. Bellal H, Rahul D, Mahadi H (2020) Zeonex-based Tetra-rectangular Core-photonic Crystal Fiber for $\mathrm{NaCl}$ Detection. Nanoscience \& $\quad$ Nanotechnology-Asia $10: 9$. doi:http://dx.doi.org/10.2174/2210681210999200708141725

19. Hossain MM, Talukder MA (2020) Optical Magnetism in Surface Plasmon Resonance-Based Sensors for Enhanced Performance. Plasmonics:1-8

20. Eid MM, Rashed ANZ, Bulbul AA-M, Podder E (2020) Mono-Rectangular Core Photonic Crystal Fiber (MRC-PCF) for Skin and Blood Cancer Detection. Plasmonics:1-11

21. Shephard J, MacPherson W, Maier R, Jones J, Hand D, Mohebbi M, George A, Roberts P, Knight J (2005) Single-mode midIR guidance in a hollow-core photonic crystal fiber. Optics Express 13 (18):7139-7144

22. Roberts P, Couny F, Sabert H, Mangan B, Williams D, Farr L, Mason M, Tomlinson A, Birks T, Knight J (2005) Ultimate low loss of hollow-core photonic crystal fibres. Optics express 13 (1):236-244

23. Pryor RW (2009) Multiphysics modeling using COMSOL®: a first principles approach. Jones \& Bartlett Publishers,

24. Pepper DW, Heinrich JC (2017) The finite element method: basic concepts and applications with MATLAB, MAPLE, and COMSOL. CRC press,

25. Islam MS, Sultana J, Atai J, Abbott D, Rana S, Islam MR (2017) Ultra low-loss hybrid core porous fiber for broadband applications. Applied Optics 56 (4):1232-1237

26. Bulbul AA-M, Imam F, Awal M, Mahmud M (2020) A Novel Ultra-Low Loss Rectangle-Based Porous-Core PCF for Efficient THz Waveguidance: Design and Numerical Analysis. Sensors 20 (22):6500

27. Iqbal F, Biswas S, Bulbul AA-M, Rahaman H, Hossain BM, Rahaman EM, Awal AM (2020) Alcohol sensing and classification using PCF-based sensor. Sensing and Bio-Sensing Research:100384. doi:https://doi.org/10.1016/j.sbsr.2020.100384

28. Bulbul AA-M, Rahaman H, Biswas S, Hossain MB, Nahid A-A (2020) Design and numerical analysis of a PCF-based biosensor for breast cancer cell detection in the THz regime. Sensing and Bio-Sensing Research 30:100388

29. Paul BK, Islam MS, Ahmed K, Asaduzzaman S (2017) Alcohol sensing over O+ E+ S+ C+ L+ U transmission band based on porous cored octagonal photonic crystal fiber. Photonic Sensors 7 (2):123-130

30. Ghazanfari A, Li W, Leu MC, Hilmas GE (2017) A novel freeform extrusion fabrication process for producing solid ceramic components with uniform layered radiation drying. Additive Manufacturing 15:102-112

31. Bise RT, Trevor DJ Sol-gel derived microstructured fiber: fabrication and characterization. In: Optical Fiber Communication Conference, 2005. Optical Society of America, p OWL6

32. Cubillas AM, Unterkofler S, Euser TG, Etzold BJ, Jones AC, Sadler PJ, Wasserscheid P, Russell PSJ (2013) Photonic crystal fibres for chemical sensing and photochemistry. Chemical Society Reviews 42 (22):8629-8648

33. Ebendorff-Heidepriem H, Schuppich J, Dowler A, Lima-Marques L, Monro TM (2014) 3D-printed extrusion dies: a versatile approach to optical material processing. Optical Materials Express 4 (8):1494-1504

34. Pysz D, Kujawa I, Stępień R, Klimczak M, Filipkowski A, Franczyk M, Kociszewski L, Buźniak J, Haraśny K, Buczyński R (2014) Stack and draw fabrication of soft glass microstructured fiber optics. Bulletin of the Polish Academy of Sciences Technical Sciences $62(4)$

35. Atakaramians S, Afshar S, Ebendorff-Heidepriem H, Nagel M, Fischer BM, Abbott D, Monro TM (2009) THz porous fibers: design, fabrication and experimental characterization. Optics express 17 (16):14053-14062

36. Cruz AL, Cordeiro C, Franco MA (2018) 3D printed hollow-core terahertz fibers. Fibers 6 (3):43 